\title{
Investigation and modeling on protective textiles using artificial neural networks for defense applications
}

\author{
Gurumurthy B. Ramaiah ${ }^{\mathrm{a}, *}$, Radhalakshmi Y. Chennaiah ${ }^{\mathrm{a}}$, Gurumurthy K. Satyanarayanarao ${ }^{\mathrm{b}}$ \\ a Central Silk Board, Central Silk Technological Research Institute, Ministry of Textiles, Textile Technology, B.T.M. Layout, Madivala, Bangalore 560 068, Karnataka, India \\ ${ }^{\mathrm{b}}$ Department of Electronics and Communication, University Visveshvaraya College of Engineering, Bangalore University, K.R. Circle, Bangalore 560 001, India
}

\section{A R T I C L E I N F O}

\section{Article history:}

Received 30 July 2009

Received in revised form 8 November 2009

Accepted 6 December 2009

\section{Keywords:}

Specific modulus

Specific tenacity

Kevlar 29

Fragment simulation projectile

Back-propagation neural networks

Dissipated energy

Bayesian information criterion

\begin{abstract}
A B S T R A C T
Kevlar 29 is a class of Kevlar fiber used for protective applications primarily by the military and law enforcement agencies for bullet resistant vests, hence for these reasons military has found that armors reinforced with Kevlar 29 multilayer fabrics which offer $25-40 \%$ better fragmentation resistance and provide better fit with greater comfort. The objective of this study is to investigate and develop an artificial neural network model for analyzing the performance of ballistic fabrics made from Kevlar 29 single layer fabrics using their material properties as inputs. Data from fragment simulation projectile (FSP) ballistic penetration measurements at $244 \mathrm{~m} / \mathrm{s}$ has been used to demonstrate the modeling aspects of artificial neural networks. The neural network models demonstrated in this paper is based on back propagation (BP) algorithm which is inbuilt in MATLAB 7.1 software and is used for studies in science, technology and engineering. In the present research, comparisons are also made between the measured values of samples selected for building the neural network model and network predicted results. The analysis of the results for network predicted and experimental samples used in this study showed similarity.
\end{abstract}

(c) 2009 Elsevier B.V. All rights reserved.

\section{Introduction}

Armor may be defined as a covering intended to defend against an exterior threat to the covered entity. All materials that provide ballistic protection (armoring material) are designed not only to prevent entry of the ballistic threat into the protected area, but also to deny entry to fragments of the ballistic threat and fragmentation of the armor itself. Materials that provide this protection when worn on the body must resist lethal levels of injury due to ballistic impact $[1,2]$. Hence energy is thus dissipated with each layer of armor material when the projectile penetrates. However the energy necessary for the projectile to penetrate is a function of many variables of fibre, yarn and fabric like the specific modulus, specific tenacity, density, extension to break, modulus, and tenacity [3,6,29]. A body armor basically consists of a ballistic vest [16] and plate that provide protection against bullets and fragmentation at velocities above $244 \mathrm{~m} / \mathrm{s}$ [4]. Until now, the ballistic armor had only been evaluated for its ability to provide ballistic protection. Impact protection models [7,20-22,32,40] consider several factors and are classified as empirical, semi- or quasi-empirical, engineering, simplified, analytical, semi-analytical $[9,10,15]$. In this study

\footnotetext{
* Corresponding author. Tel.: +91 80 26680435/9818970053; fax: +91 8026688831 .

E-mail address: brgmurthy@live.com (G.B. Ramaiah).
}

the problem associated with the behavior of Kevlar 29 single layer fabric due to its material properties is analyzed with reference to its performance variables like depth of penetration and amount of energy dissipated $[17,23]$. Neural network models [15] are useful from the viewpoint of their direct applicability, since they do not depend on any theoretical assumptions and material investigation results are directly used for building the model and studying the performance of ballistic protective materials. In the present study emphasis is made on investigation and building neural network models based on data collected from samples which find similar applications in protective textiles [4]. The data is also used for studying, comparing measured and network predicted ballistic test parameters which fall within the objectives of the study. This study focuses on development of neural network models using Matlab 7.1 as one of the softwares used for simulation, design and optimization of several problems and analysis of non-linear relationships between variables which are complex in nature. The results show that the neural network can be successfully used in modeling based on material properties of Kevlar 29 ballistic single layer fabrics and hence give an opportunity to explore new ideas of understanding the performance criterion of armor protective textiles.

\section{Theoretical study}

Pressure waves can injure neural cells $[9,10,15]$. However, the physical mechanisms by which impact pressure waves reach the 
brain and cause injury are not well understood. Several theories, which are not mutually exclusive, have been suggested [5,11-14]. Possibilities include acceleration of the head, direct passage of the blast wave via the cranium, and propagation of the blast wave to the brain via a thoracic mechanism. It is important to determine how impact pressure waves are transmitted to the brain so that exposure risks can be assessed and so effective preventive measures can be implemented [24,38]. Many theoretical models are been proposed, a simple estimate of the peak pressure ( $p$ in $\mathrm{kPa}$ ) at a distance $(r$ in $\mathrm{cm})$ from the bullet path is given by

$p \approx \frac{10 E}{d 4 \pi r^{2}}$

where $E$ is the kinetic energy (joules) of the bullet (mass times the square of the speed), and $d(\mathrm{~cm})$ is the penetration distance $[18,31]$. Note that the magnitude of the pressure wave decreases as the square of the distance from the bullet. While the acoustic impedances of water and soft tissues are similar, the exact transfer function for the pressure wave is not known and will vary to some extent with the animal model used. Therefore, preliminary tests must be performed to verify actual pressures in the specific experimental design. Another simple model behind armor mechanical work, $W$, as a function of armor deformation, $d$, is given by

$W(d)=E \times\left(1-\frac{1}{1+\left(d / d_{0}\right)^{3}}\right)$,

where $E$ is the impact energy (joules) of the bullet, and $d_{0}(\mathrm{~cm})$ is an adjustable parameter that sets the armor deformation depth at which half of the impact energy is available to do work creating forces and pressures in behind armor tissues. This model is physically reasonable (2). It has the expected monotonic and limiting behaviors, giving zero work for body armor which stops the projectile with zero armor deformation and approaching the maximum possible work as the armor deformation becomes very large [25].

\subsection{Effect of fabric structural variables on prediction model}

The rate of energy absorption of Kevlar 29 ballistic protective fabric not only depends on the fibre modulus which increases monotonically, but also on the material modulus and its braking strain $[19,33]$. In case of layered woven panels used as protective vests, each fibre crossovers acts to reflect a portion of the stress wave which is propagated through different layers. The modulus of each fabric in the panel controls wave speed, and thus the distance the impact disturbance will have travelled in a given time. The modulus also controls the level of strain, which will be generated by impact at a given velocity. These bulletproof properties are determined by the characteristics of fibre such as strength, elongation, modulus, and rupture. Additionally, the characteristics of fabric such as its structure, thickness, and the number of layers also affect the bulletproof properties. The breaking energy of the fibre and the transmitting velocity of the stress wave determine the property of the material that is used for impact resistance. The stress wave needs to be dispersed rapidly, and the breaking energy needs to be increased in order to raise the impact resistance [33]. The breaking energy of the material is its ability to resist the breaking of by an external force, and is a function relative to the tensile strength and the elongation. The ability of the material to absorb energy is better with multiple layers, while the tensile strength and the deformation capacity are greater in theory for layered fabrics.

\section{Artificial neural network modelisation}

Previous study [4] on Kevlar 29 composites indicates enhanced material properties for ballistic penetration resistance in the fabric after treating with colloidal shear thickening fluids (STFs) $[35,36]$ under conditions of low velocities and small target sizes. However a study on the properties of Kevlar 29 single layer fabrics using artificial neural networks is a new idea which is explored in this paper from the viewpoint of building a predictive model using material properties (single layer fabrics) [19] and their ballistic fabric performance data. Regression models (such as artificial neural networks) have been proposed by Weaver [10]. However in his study he employs nested neural networks (NNNS) to develop the regression model. Case studies of using NNNS indicate the robustness of his methodology applied to study ballistic missile defense systems (BMDS).

\subsection{Data collection and processing}

The most commonly used materials according to the National Institute of Justice (NIJ guide 100-01) [2] are marketed under the names Kevlar ${ }^{\circledR}$, Spectra ${ }^{\circledR}$, Dyneema ${ }^{\circledR}$, and Zylon ${ }^{\circledR}$. DuPont's Kevlar 29 fiber was the first material used in modern concealable body armor $[27,28,43,44]$. Kevlar 29 in general is an organic manmade aramid fiber that is flame resistant, does not melt, and has high strength combined with low weight. Kevlar 29 also has high chemical resistance and cut resistance (DuPont, 2001). Kevlar 29 single layer fabrics used for armor applications are the materials used in this study and data of these fabrics are considered and collected from experiments of ballistic tests and processed for artificial neural network modelisation.

\subsection{Sample selection for artificial neural network modeling}

One of the most critical issues when using neural networks is how to select appropriate samples and network architectures for the problem at hand [47]. If the sample information criterion is not clear than this might lead to over-parameterized models with heavy consequence on overfitting and poor ex post forecast accuracy. Moreover, since model selection criteria depend on sample information, their actual values are subject to statistical variations. Here the sample information is subjected to statistical tests like mean and standard deviation for retaining accuracy of the models at its input level itself. This is done to avoid the possibility of any satisfactory results obtained which may simply be due to chance rather than any merit inherent in the model yielding to the result. Bayes factor information [39] on modeling provides sample information on the optimum number of hidden neurons where generalizations are found to be good [42]. The sample data and their information representing true population is shown in Table 1.

\subsection{Artificial neural network modeling procedure}

Artificial neural networks (ANNs) have become a powerful tool for many complex applications such as function approximation, optimization, non-linear system identification and pattern recognition. This is due to the fact that they are capable to learn from examples and to perform non-linear mappings. It is confirmed that neural networks can reduce considerably the task of engineers by allowing an effective generic approach of non-linear problems. The back-propagation has been applied to a wide variety of practical problems and it has proven very successful in its ability to analyze non-linear relationships. A simple back-propagation neural net is shown in Fig. 1.

Data are used to develop the model. Before modeling the data base is divided into three parts (sets), that is, training set, validation set and test set. The training set is used to train the model, the validation set is used to validate the model in order to prevent the model from overfitting, and the test set is used to test the model in order to select an accurate model. 

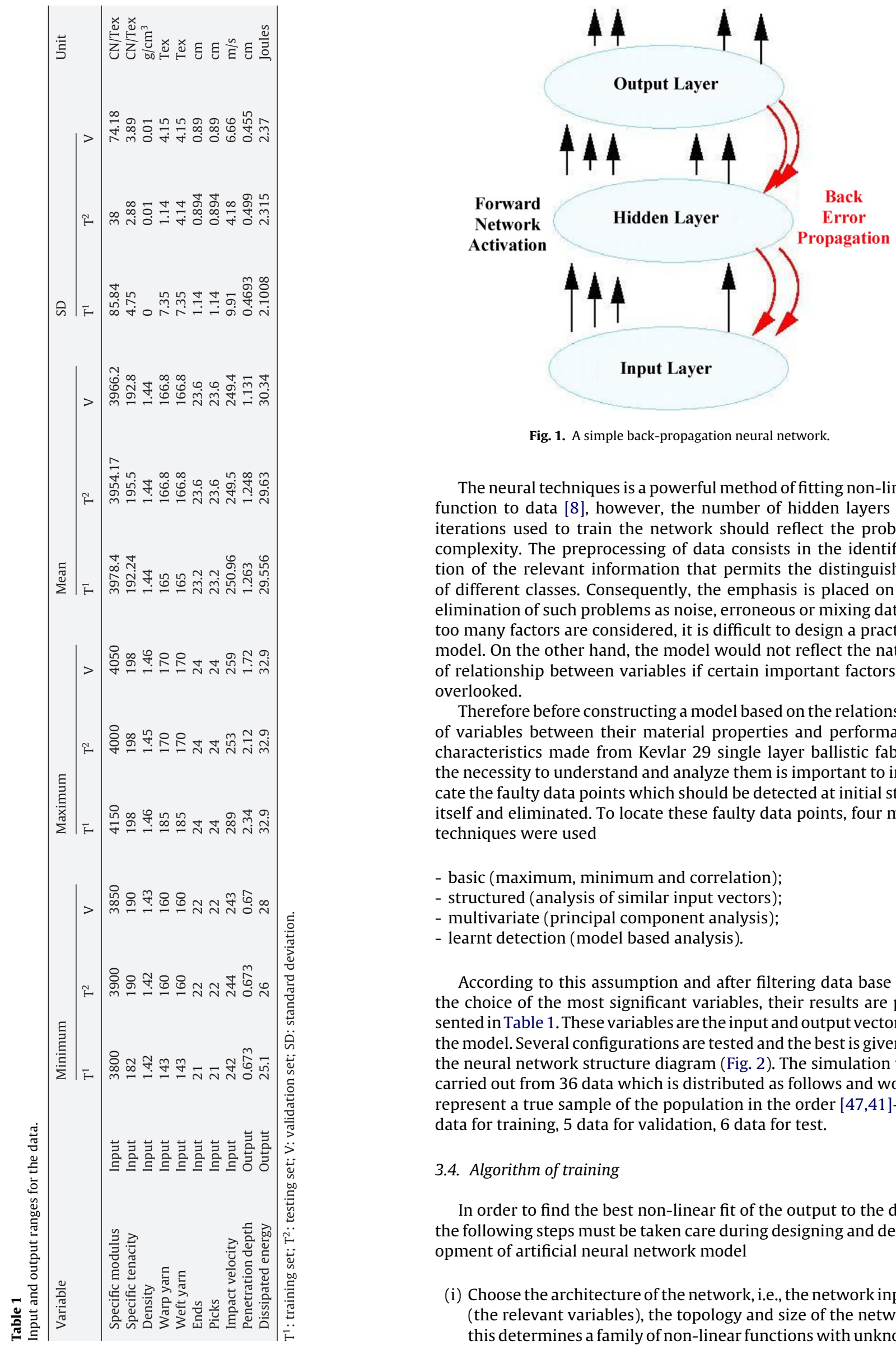

Fig. 1. A simple back-propagation neural network.

The neural techniques is a powerful method of fitting non-linear function to data [8], however, the number of hidden layers and iterations used to train the network should reflect the problem complexity. The preprocessing of data consists in the identification of the relevant information that permits the distinguishing of different classes. Consequently, the emphasis is placed on the elimination of such problems as noise, erroneous or mixing data. If too many factors are considered, it is difficult to design a practical model. On the other hand, the model would not reflect the nature of relationship between variables if certain important factors are overlooked.

Therefore before constructing a model based on the relationship of variables between their material properties and performance characteristics made from Kevlar 29 single layer ballistic fabrics the necessity to understand and analyze them is important to indicate the faulty data points which should be detected at initial stage itself and eliminated. To locate these faulty data points, four main techniques were used

- basic (maximum, minimum and correlation);

- structured (analysis of similar input vectors);

- multivariate (principal component analysis);

- learnt detection (model based analysis).

According to this assumption and after filtering data base and the choice of the most significant variables, their results are presented in Table 1. These variables are the input and output vectors of the model. Several configurations are tested and the best is given by the neural network structure diagram (Fig. 2). The simulation was carried out from 36 data which is distributed as follows and would represent a true sample of the population in the order $[47,41]-25$ data for training, 5 data for validation, 6 data for test.

\subsection{Algorithm of training}

In order to find the best non-linear fit of the output to the data, the following steps must be taken care during designing and development of artificial neural network model

(i) Choose the architecture of the network, i.e., the network inputs (the relevant variables), the topology and size of the network, this determines a family of non-linear functions with unknown 


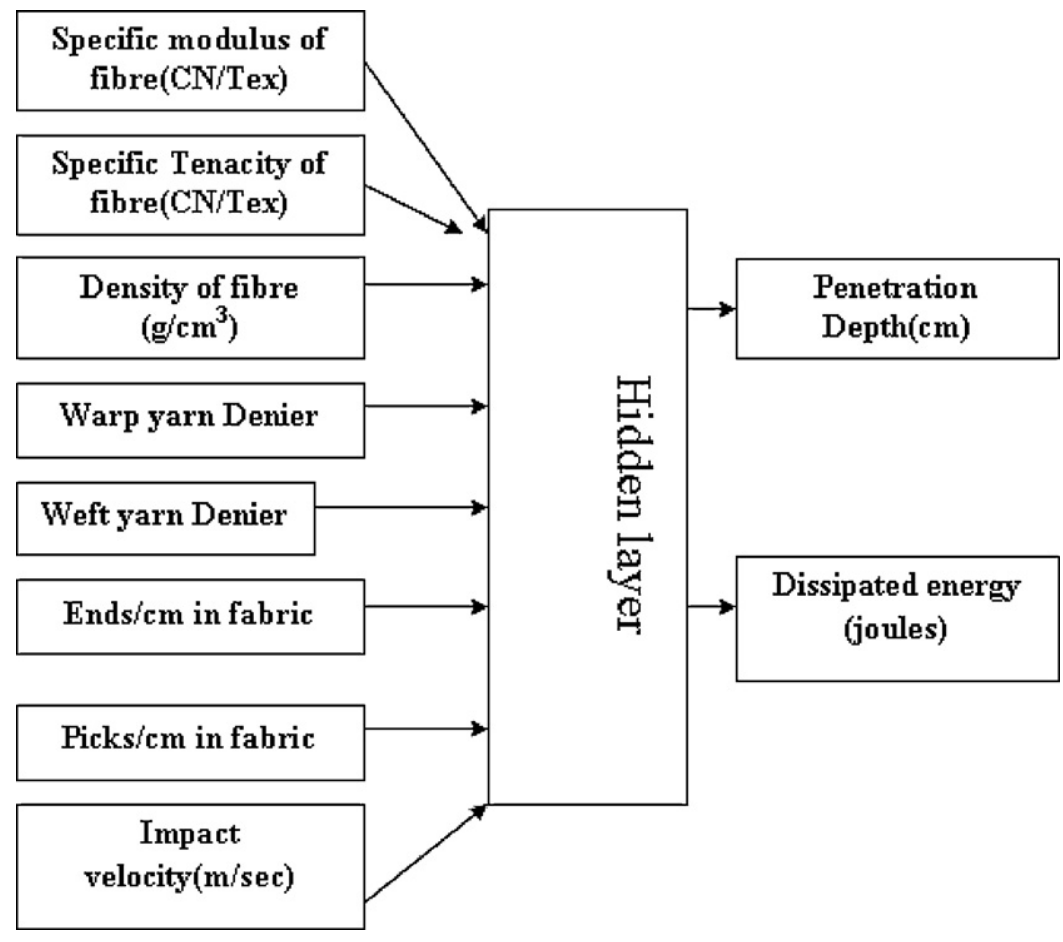

Fig. 2. Neural network model for Kevlar 29 ballistic fabric.

parameters (the weights of the network) which are candidates for performing the data fitting.

(ii) Train the network, i.e., compute the set of weight values that minimize the approximation error over the data set used for training (training set).

(iii) Assess the performance of the network on a data set (called test set) which is distinct from the training set, but which stems from the same population.

The back-propagation is the popular algorithm used in practice. The network is called back-propagation because the errors in the network are fed backward, or back propagated, through the network (Fig. 1). This model has been adapted to analyze relationships between ballistic fabrics in the present study, and has found lots of applications in various domains of science, technology and engineering. Back-propagation is typically done on feed forward neural networks and is able to generalize well on a wide variety of problems [37]. These training methods are called supervised training because they are trained with both inputs and outputs. Input signals propagate through the network layer by layer, in the end producing a response at the output of the network. This phase of the operation of back-propagation is called the forward phase. The output of the network is compared with the target response, generating error signals. These error signals propagate in a backward direction through the network. In this phase, the weights of the network are adjusted to minimize the sum of squared errors:

$E=\frac{1}{2} \sum_{i}\left(t_{i}-y_{i}\right)^{2}$

where $t_{i}$ is the $i$ th desired output (target) and $y_{i}$ is the $i$ th output. The weights were updated using delta function and each hidden layer was appropriately updated with activation function. In this study we exposed the training data to the neural network with epoch based training and the weights were updated automatically. The architecture of the optimized neural network consisted of 8 inputs, 4 hidden neurons, and 2 outputs neurons. The input and output layers consisted of neurons with linear activation functions:

$\phi(v)=v$

The hidden layer consisted of sigmoid activation functions

$\phi(v)=\frac{1}{1+e^{-a v}}$

where $a$ is the slope parameter of the sigmoid activation function.

Conjugate-gradient descent methods of training algorithm is used which has a second-order convergence property without complex calculation of the Hessian matrix. A faster convergence is expected than other approaches. Conjugate-gradient approach finds the optimal weight vectors along the current gradient by doing a line-search. It computes the gradient at the new point and projects it onto the subspace defined by the complement of the space defined by all previously chosen gradients. The new direction is orthogonal to all previous search directions. The method is simple. No parameter is involved. It requires little storage space and expected to be efficient [39].

\subsection{Model variables}

The various model variables used for artificial neural network modelisation included in this study are shown in Fig. 2 [29]

(i) Specific modulus (CN/Tex)-Specific modulus is a materials property consisting of the elastic modulus per mass density of a material. It is also known as the stiffness to weight ratio or specific stiffness. High specific modulus materials find wide application in aerospace applications where minimum structural weight is required.

(ii) Specific tenacity ( $\mathrm{CN} / \mathrm{Tex}$ )-Force required to break the fabric during tensile testing and is determined using tensile testing machines is called tenacity. Specific tenacity is the force divided by the linear density of the material at break. 
Correlation chart for Penetration depth-Testing set

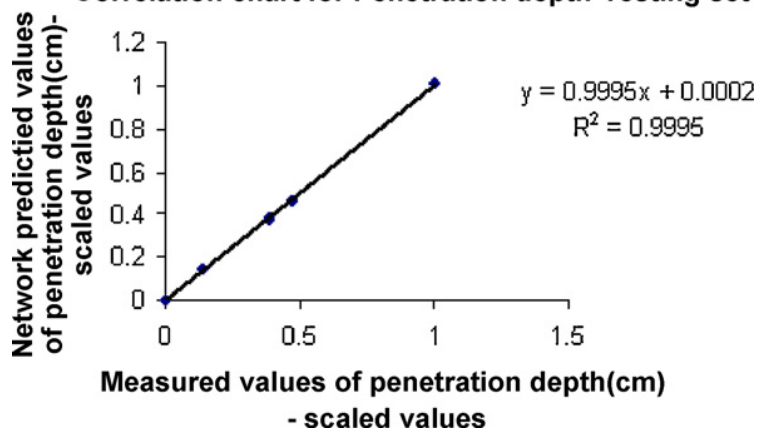

Fig. 3. Comparison between measured and network values of penetration depth (cm).

(iii) Density $\left(\mathrm{g} / \mathrm{cm}^{3}\right)$-Fiber density is the ratio of mass to volume of the fiber. Fiber density is determined by using a Psychometer, density balance (Archimedes principle) or Density gradient method. ASTMD3800 gives Archimedes and sink/float method of determining fiber density where as ASTM D1505 gives the determination of fiber density using density gradient column methods.

(iv) Impact velocity $(\mathrm{m} / \mathrm{s})$-The velocity of a projectile or missile at the instant of impact. This is also known as striking velocity $(\mathrm{m} / \mathrm{s})$.

(v) Penetration depth $(\mathrm{cm})$-Penetration depth is a measure of how deep the projectile or missile can penetrate into a material. This is usually measured in cms.

(vi) Dissipated energy-This is the energy that is dissipated through the protective cloth layers due to the impact of the projectile or missile. This is measured in joules.

\section{Results and discussion}

After training, the capacity of the network is examined using other data in the so-called test set [47]. The network is presented by new examples which also contain output information (as in training). The procedure corresponds to that of the training phase, but the data are not used for learning. In this test, the most important information is the error generated by the network. If this is considered being too large, training should be continued. If the results achieved by the network are satisfactory, it can be used for problem solving. The data used here no longer contain desired output values. They now consist of the input information only. This action on the network is called test. Optimal values found for momentum factor and learning rate are 0.3 and 0.1 [42,29,30]. A further important consideration is the performance of the neural technique in comparison with the measured results. Figs. 3 and 4 indicate performance of the prediction model compared between predicted and measured values which show excellent correlation.

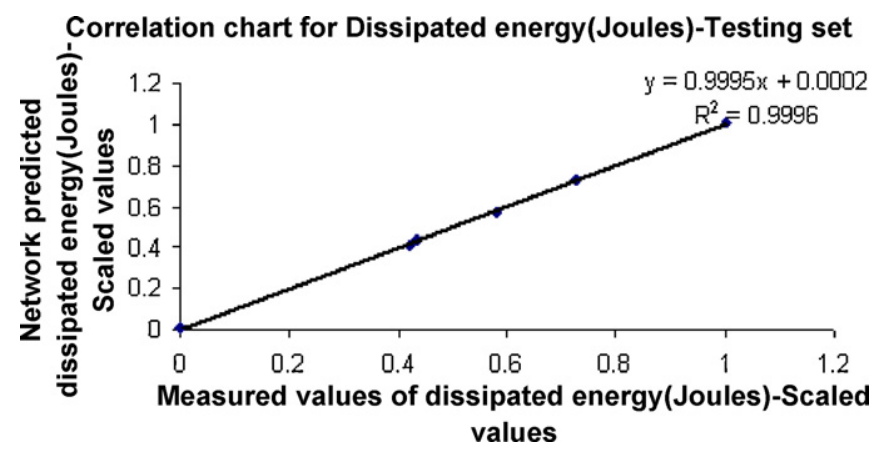

Fig. 4. Comparison between measured and network values of dissipated energy (joules).
Network architecture configuration $8-4-2$ is chosen after carefully observing Bayesian factors like number of effective parameters and error terms from the optimization studies carried out on the model. The prediction models and its errors on the test data confirm the reliability of the methodology followed. The output parameters of training with Bayesian regularization [39] with respect to epoch number indicated that training of neural networks can be achieved quickly. The errors and Bayesian information criterion reported during validation tests of model were sum squared error (SSE) 1.775 and effective number of parameters is 1.606 with the neural network model being validated at 15 epochs.

The present model build from material properties like specific modulus, specific tenacity, density of fibers, yarn tex used to make the fabric along with their thread density and impact velocity as inputs and penetration depth coupled with dissipated energy as performance parameter of neural network model promises excellent results [29] which can be used for forecasting performance properties of Kevlar 29 ballistic fabrics provided the underlying forces driving the variation in those data remain reasonably constant over time [45-47]. They are useful as an alternative to more conventional statistical tools in exploring the prognostic information in a performance evaluation data set [42]. They are likely to be useful to expert analyst in their assessment and design of ballistic textiles in general $[19,26,34]$. However the proposed model does not take into account the variability of ballistic performance properties and material properties arising due to effect of different fabrics layers which need to be addressed with different set of test data and in particular to take address the issues of the composite layer as a whole [25].

\section{Conclusion}

The objective of this study is to develop a model based on backpropagation neural networks for predicting dissipation energy and penetration depth during firing and resistance offered by bullet proof vests (single layer) made of Kevlar 29 fibers. The developed prediction model in this study gives excellent correlations with the samples selected. The optimized networks can be used for design, development of ballistic protection fabrics without going for further destructive tests. This therefore represents a very significant development and the performance of the developed model which is strengthened with Bayesian information criterion coupled with gradient descent algorithm would overcome the problem of determining optimum number of neurons in hidden layer and shows satisfactory results. A mixed neural network would create a knowledge base on current materials and satisfactory research is possible by studying closely the new designs which would be much better than the existing ones. The present study proposes to overcome the limitations of conventional fiber science and theoretical models used for analysis by providing opportunity to analyze the properties of ballistic fabrics using newer techniques and methods of simulations relating to their structure behavior during ballistic tests. Overall the results show that the neural network can be successfully used in modeling material properties of ballistic fabrics with similarity and better accuracy compared to traditional fibre science methods of study.

\section{Acknowledgements}

The authors wish to thank the CSIR-India, Central Silk Board/Central Silk Technological Research Institute, Ministry of Textiles, Government of India for providing financial assistance to carry out research work in the area of artificial neural networks and research work on textiles under their expert guidance. 


\section{References}

[1] C.R.D. Bass, K.A. Rafaels, R.S. Salzar, M. Carboni, R.W. Kent, M.D. Lloyd, S. Lucas K. Meyerhoff, C. Planchak, A. Damon, G.T. Bass, Journal of Accident Analysis \& Prevention 40 (2) (2008) 487-495.

[2] M.J. Waters, Laboratory Test Methods for Evaluating Protective Clothing Systems Against Chemical Agents, US Army CRDC-SP-84010, 1984.

[3] R.V. Iyer, V. Kalyani, K. Sooryanarayana, Row, Bulletin of Materials Science 22 (1) (2008) 1-7.

[4] E.D. Wetzel, Y.S. Lee, R.G. Egress, K.M. Kirkwood, J.E. Kirkwood, N.J. Wagner, Journal of Material Science 38 (13) (2003) 2825-2833.

[5] G.B. Kingston, H.R. Maier, M.F. Lambert, Journal of Hydrology 314 (1-4) (2005) $158-176$.

[6] H.S. Shin, D.C. Erlich, D.A. Shockey, Journal of Materials Science 38 (2003) 3603-3610.

[7] G.B. Kingston, H.R. Maier, Mathematical and Computer Modelling 44 (5-6) (2006) 499-512.

[8] M. Lefik, D.P. Boso, B.A. Schrefler, Computer Methods in Applied Mechanics and Engineering 198 (21-26) (2009) 1785-1804.

[9] P.M. Cunniff, Textile Research Journal 66 (1) (1996) 45-58.

[10] B.L.Weaver, A methodology for Ballistic missile defense systems analysis using nested neural networks, Thesis submitted to Academic Faculty, School of Aerospace Engineering, Georgia Institute of Technology, 2008.

[11] A. Courtney, M. Courtney, Medical Hypotheses 72 (1) (2008) 76-83.

[12] A.A. Cerit, M.B. Karamis, F. Nair, Tribology in Industry $26(3,4)(2004)$.

[13] A. Weinberg, P. Schwartz, Journal of Materials Science Letters 6 (1987) 183-184.

[14] M.H. Lafitte, A.R. Bunsell, Journal of Material Science 17 (8) (1982) 2391-2397.

[15] K. Shihab, Journal of Computer Science 2 (9) (2006) 710-715.

[16] B.J. Briscoe, F. Motamedi, Wear 158 (1-2)(1992) 229-247.

[17] V.B.C. Tan, T.W. Ching, International Journal of Impact Engineering 32 (11) (2006) 1737-1751.

[18] Y. Duan, M. Keefe, T.A. Bogetti, B.A. Cheeseman, B. Powers, International Journal of Impact Engineering 32 (8) (2006) 299-1312.

[19] Y. Duan, M. Keefe, T.A. Bogetti, B.A. Cheeseman, International Journal of Impact Engineering 31 (8) (2005) 996-1012.

[20] A. Shahkarami, R. Vaziri, International Journal of Impact Engineering 34 (1) (2007) 104-119.

[21] A. Peled, A. Bentur, Cement and Concrete Research 30 (5) (2000) 781-790.

[22] D.A. Powell, T.I. Zohdi, Composites Part B: Engineering 40 (6) (2009) 451-460.

[23] C.T. Lim, V.P.W. Shim, Y.H. Ng, International Journal of Impact Engineering 28 (1) (2003) 3-31.
[24] B. Nadler, P. Papadopoulos, D.J. Steigmann material, International Journal of Solids and Structures 43 (2) (2006) 206-221.

[25] C.M. Leech, B.A. Adeyefa, Computers \& Structures 15 (4) (1982) 423-432.

[26] R.H. Zee, C.Y. Hsieh, Materials Science and Engineering A 246 (1-2) (1998) 161-168.

[27] V.P.W. Shim, V.B.C. Tan, T.E. Tay, International Journal of Impact Engineering 16 (4) (1995) 585-605.

[28] M.P. Rao, Y. Duan, M. Keefe, B.M. Powers, T.A. Bogetti, Composite Structures 89 (4) (2009) 556-566.

[29] N. Pan, Composites Science and Technology 56 (3) (1996) 311-327.

[30] N.K. Naik, P. Shrirao, Composite Structures 66 (1-4) (2004) 579-590.

[31] N.K. Naik, P. Shrirao, B.C.K. Reddy, International Journal of Impact Engineering 32 (9) (2006) 1521-1552.

[32] B. Parga-Landa, F.H. Olivares, International Journal of Impact Engineering 16 (3) (1995) 455-466.

[33] D. Roylance, S.S. Wang, Fibre Science and Technology 14 (3) (1981) 183-190.

[34] B.A. Cheeseman, T.A. Bogetti, Composite Structures 61 (1-2) (2003) 161-173.

[35] D. Sadagopan, R. Pitchumani, Composites Science and Technology 58 (3-4) (1998) 571-589.

[36] T.I. Zohdi, D. Powell, Computer Methods in Applied Mechanics and Engineering $195(1-3)(2006) 94-109$

[37] B. Zhang, D.S. Sikes, C. Muster, S.Q. Li, System Biology 57 (April (2)) (2008) 202-215

[38] S. Lawrence, C. Lee Giles, IEEE-INNS-ENNS International Joint Conference on Neural Networks (IJCNN'00), vol. 1, 2000, p. 1114.

[39] D.J.C. MacKay, Networks Neural Computation 4 (3) (1992) 448-472.

[40] D.A. Winkler, F.R. Burden, Journal of Molecular Graphics and Modelling 22 (6) (2004) 499-505.

[41] B. Schenker, M. Agarwal, Computers \& Chemical Engineering 20 (2) (1996) $175-186$.

[42] H. Meradi, S. Bouhouche, M. Labreche, Engineering and Technology 24 (2006) $120-124$.

[43] N.K. Naik, P. Shrirao, B.C.K. Reddy, Materials Science and Engineering: A 412 (1-2) (2005) 104-116.

[44] S.S. Morye, P.J. Hine, R.A. Duckett, D.J. Carr, I.M. Ward, Composites Science and Technology 60 (14) (2000) 2631-2642.

[45] L. Tarassenko, Data, Guide to Neural Computing Applications, 1998, pp. 67-75.

[46] A. Heiat, Information and Software Technology 44 (15) (2002) 911-922.

[47] A. Forcellese, F. Gabrielli, R. Ruffini, Journal of Materials Processing Technology 80-81 (1998) 493-500. 